\title{
Humanized Artificial Intelligence
}

\author{
Matthew N. O. Sadiku', Yogita P. Akhare', Abayomi Ajayi-Majebi ${ }^{1}$, and Sarhan M. Musa ${ }^{1}$ \\ ${ }^{1}$ Roy G. Perry College of Engineering \\ Prairie View A\&M University \\ Prairie View, TX 77446 \\ ${ }^{2}$ Department of Manufacturing Engineering \\ Central State University \\ P.O. Box 1004 \\ Wilberforce, $\mathrm{OH}$ 45384-1004
}

\begin{abstract}
Artificial Intelligence (AI) is intelligence demonstrated by a machine, as opposed to human intelligence. The field of artificial intelligence is based on the premise that human intelligence can be so precisely described and simulated by a machine. Evidence of the positive impact of AI systems is all around us. It will never be possible for such machines to completely replace human beings partly due to the fact that AI lacks a human touch. Organizations and individuals around the world are creating core principles around AI with an emphasis on a more humanist approach. They realize the significant advantages AI can bring to their business. This paper provides an introduction to humanized AI.
\end{abstract}

Key Words: Artificial Intelligence, Artificial Intelligence Humanized AI, Human AI System.

\section{INTRODUCTION}

Intelligence refers to the mental ability for reasoning, problem-solving, and learning. It has the capabilities to learn from past experience, adapt to new situations, and handle abstract ideas. It can be measured by standardized tests and used to determine educational achievement, job performance, and health conditions.

Human beings have the natural ability to sense their environment, interpreting what they see, responding to stimuli, and empathizing with the environment and with other fellow human beings. However, humans are limited in terms of computing high numbers and repetitive tasks. Artificial Intelligence (AI) can extend the skills of humans by learning to perform and automate tasks in manners designed by humans. AI performs tasks in a robotic manner. Although AI can provide massive computational powers, it is yet to handle the concept of emotions like happiness, sadness, depression, stress, anger, and pain. This is evident in the case of wealth management, where a human expert, rather than an AI counterpart, is received well by the customer. However, AI improves its ability to empathize only with the availability of data. It requires a lot of intimate information about an individual to be able to truly understand the person's physical or mental limitations. Research on AI employs different tools from many fields, including computer science, psychology, philosophy, logic, neuroscience, cognitive science, linguistics, operations research, economics, control theory, and probability.

\section{OVERVIEW ON ARTIFICIAL INTELLIGENCE}

The term "artificial intelligence" (AI) was coined in 1956 by John McCarthy during a conference held on this subject. AI is the branch of computer science that deals with designing intelligent computer systems that mimic human intelligence. It is sometimes called machine intelligence, intelligence demonstrated by machines, in contrast to the natural intelligence displayed by humans. The ability of machines to process natural language, to learn, to plan makes 
it possible for new tasks to be performed by intelligent systems. The main purpose of AI is to mimic the cognitive function of human beings and perform activities that would typically be performed by a human being. AI is a standalone independent electronic entity that functions much like a human expert. Today, AI is integrated into our daily lives in several forms, such as personal assistants, automated mass transportation, aviation, computer gaming, facial recognition at passport control, voice recognition on virtual assistants, driverless cars, companion robots, etc. [1].

An important feature of AI technology is that is can be added to existing technologies. AI has benefited many areas such as chemistry and medicine, where routine diagnoses can be initiated by AI-aided computers. It embraces a wide range of disciplines such as computer science, engineering, machine learning, chemistry, biology, physics, astronomy, neuroscience, and social sciences.

$\mathrm{AI}$ is not a single technology but a range of computational models and algorithms. The major disciplines in AI include expert systems, fuzzy logic, and artificial neural networks (ANNs), machine learning, deep learning, natural language processing, computer vision, and robotics. The various computer-based tools or technologies that have been used to achieve AI's goals are the following [2,3]:

- Expert Systems: An expert system (ES) (or knowledge-based system) enables computers to make decisions by interpreting data and selecting between alternatives, just as a human expert would do. It uses a technique known as rule-based inference, in which rules are used to process data.

- Neural Networks: These computer programs identify objects or recognize patterns after having been trained. Artificial neural networks (ANNs) are parallel distributed systems consisting of processing units (neurons) that calculate some mathematical functions. The ANN model represents nonlinear relationships that are directly learned from the data being modeled. Neural networks are being explored for healthcare applications in imaging and diagnoses, risk analysis, lifestyle management and monitoring, health information management, and virtual health assistance.

- Natural Language Processors: Computer programs that translate or interpret language as it is spoken by normal people. NLP techniques extract information from unstructured data to supplement and enrich structured data. NLP targets extracting useful information from the narrative text to assist clinical decision making. NLP includes applications such as speech recognition, text analysis, translation and other goals related to language. There are two basic approaches to NLP: statistical and semantic. Healthcare is the biggest user of NLP tools [4].

- Robots: Computer-based programmable machines that have physical manipulators and sensors. The introduction of intelligent robots in the healthcare domain enhances patients' satisfaction, the accuracy of diagnosis, and the operational efficiency of hospitals. Robotic guidance is becoming common in spine surgery [5].

- Fuzzy Logic: Reasoning based on imprecise or incomplete information in terms of a range of values rather than point estimates. Fuzzy logic deals with uncertainty in the knowledge that simulates human reasoning in incomplete or fuzzy data. The fuzzy model is robust to parameter changes and tolerant to impression.

- Machine Learning: Algorithms to make predictions and interpret data and "learn", without static program instructions. ML is a statistical technique for fitting models to data and training models with data. ML extracts features from input data by constructing analytical data algorithms and examines the features to create predictive models. The most common ML algorithms are supervised learning, unsupervised learning, reinforcement learning, and deep learning. The most common application of ML is precision medicine. ML algorithms are capable of identifying suicide risk factors.

- Deep Learning: A subset of machine learning built on a deep hierarchy of layers, with each layer solving different pieces of a complex problem. It aims at increasing the capacity of supervised and unsupervised learning algorithms for solving complex real-world problems by adding multiple processing layers [6].

- Data Mining: This deals with the discovery of hidden patterns and new knowledge from large databases. Data mining exhibits a variety of algorithmic tools such as statistics, regression models, neural networks, fuzzy sets, and evolutionary models.

Each AI tool has its own advantages. Using a combination of these models, rather than a single model, is recommended. AI technologies are drastically influencing the retail industry and customer experience. 


\section{CONCEPT OF HUMANIZED AI}

Humanized AI attempts to create AI that is more human. It is a call for building human systems where the efficiency, programs, processes, and outcomes can be assessed and improved on the basis of data. It envisions the state of the world where humans and machines work together, each leveraging its comparative advantages with humans in the drivers' seat. Various attempts have been made to integrate fundamentally human ideas like judgment, empathy, or fairness into an AI equation.

Whatever affects humans requires a humanized approach. AI is no exception. Humanized AI is that which understands human emotions like happiness, stress, urgency, anger to detect emotions like laughter, anger, arousal, and pain. It responds to natural language very much like a human friend.

Perhaps a good way to understand humanized AI is to understand human intelligence. Humans have the following characteristics [7]:

1. Limited memory: Humans have limited memory, and sometimes, even when faced with the exact same situation than previously encountered. They use memory and thinking, while robots use built-in instructions, designed by engineers and scientists.

2. Limited processing: When problems size grows, strong rational skills are required, and humans are limited. However, human beings can work on multiple responsibilities.

3. Emotions: Humans have emotions. Emotions are probably the most impacting factor for human decisionmaking.

4. Environment: To feel human involves feeling secure and familiar with our surroundings, both physical and culturally. Humans have the ability to change their own environment using the gained knowledge. They can adapt to the environment using a combination of several cognitive processes. Social context often defines appearance through cultural influences.

5. Creativity: Creativity and imagination are the main characteristics of the human species. Human creativity is superior to AI because it is the creation of God. By definition, artificial intelligence is artificial, little, and temporarily created by humans.

In contrast to human intelligence, artificial intelligence has the following advantages [5]

- Speed of execution: For example, a doctor can make a diagnosis in 10 minutes, AI system can make a million for the same time.

- Less Biased: AI systems do not involve emotions or biased opinions on the decision-making process

- Operational Ability: They do not expect a halt in their work due to saturation

- Accuracy: Preciseness of the output increases

Artificial Intelligence can beat human intelligence in some specific areas. Let us consider three examples. First, in chess, a supercomputer has beaten the human player. Second, AI technologies such as IBM Watson are being used at some medical centers to support diagnosis and create man. Third, Sophia is a social robot created by former Disney Imagineer David Hanson. Sophia behaves like a human. The robot has sat for TV interviews, appeared on the cover of ELLE magazine, been parodied on HBO, and was appointed the UN's first non-human "innovation champion." The guiding principle of $\mathrm{AI}$ is not to become autonomous or replace human intelligence.

\section{APPLICATIONS}

Humanization reflects on those things which make us feel more human. Humanizing principles can be applied to every machine that involves human-AI collaboration [8]. Common areas in which humanized AI is currently observed include education, healthcare, and financial services, which affect human life directly, socially, physically, and economically [9].

- Healthcare: One of the greatest challenges faced by healthcare professionals is providing humanized care that is acceptable in current medical practices. Using neural network, AI algorithm can identify and categorize the level of depression through a study of characteristics of speech, amount of breath, word choice, etc. Healthcare professionals should rethink their behaviors when providing care to patients so they can ensure quality care based on humanization [10]. Figure 1 shows an example of the Human-AI system in healthcare [11]. 
- Financial Services: Several financial multi-national companies are experimenting with AI in finance to bring more benefits to customers. Financial services industries have tended to build more secure systems as people's money is at stake. Companies are using AI principles to leap ahead on innovation and profitability.

- Education: AI and personalization go hand in hand. Personalized learning through analytics and artificial intelligence has been enabled for several classrooms by pioneers. AI can be used to engage with the students in solving problems. Education will be crucial in the 21 st century and may need to be redesigned to meet the challenges.

\section{BENEFITS AND CHALLENGES}

A lot of companies benefit from how powerful and scalable their AI is. AI is capable of fighting fraud that affects consumers. AI systems have significantly taken over many tasks, especially when it comes to monotonous judgments. The utilization of AI will make life more convenient for humankind. AI systems would be able to express a mixture of feelings, such as fear, doubt, hope, as anger, revenge, trust, mistrust, empathy, etc., as an integral part of human behavior.

For the sake of privacy, security, transparency, and ethics, we need to be thoughtful about how AI technology can be used, where it can be used, when it can be used, and what it can access. Not addressing these privacy and security questions will hinder the adoption of AI. The more we train machines and expect them to emulate us as social beings, the more we are confronted with the same ethical issues that accompany human interactions [12]. Some are concerned about the fate of humankind in the "fourth industrial revolution" and the emergence of artificial intelligence and big data and AI. Human-AI collaboration has cultural and social implications. For example, should the driverless car kill elderly people if it has no choice? Today's AI systems are still dependent on human input and not completely autonomous. It is crucial for an AI to simulate human-like learning, decision making, and most importantly, autonomy [13]. This will increase confidence in their decision-making.

\section{CONCLUSION}

The advances in AI are reaching new peaks with unprecedented speed. As AI technologies continue to impact our daily lives, it is expected that AI systems will work synergistically with humans. Organizations and people around the world are building trustworthy and dependable artificial intelligence. Business leaders are getting significant value from advanced $\mathrm{AI}$ in their companies. $\mathrm{AI}$ is critical to the business of the future.

Although experts claim that the rise of networked artificial intelligence will make most people better off with time, many are concerned about how advances in AI will affect what it means to be human, to be productive, and to exercise free will. The AI algorithms of machines simply extend human capabilities and not replace them. A human AI is a human social system that would apply and leverage the power of data and the principles of AI. It is hoped that human systems would become better off, safer, fairer, more civil, and more sustainable with time [14]. Intelligent machines are the future of humanity [15]. For more information about human-AI systems, one should consult the books in [1617].

\section{REFERENCES}

[1] Y. Mintz and R. Brodie, "Introduction to artificial intelligence in medicine," Minimally Invasive Therapy \& Allied Technologies, vol. 28, no. 2, 2019, pp. 73-81.

[2] R. O. Mason, "Ethical issues in artificial intelligence," Encyclopaedia of Information Systems, vol 2, 2003, pp. 239258.

[3] A. N. Rames et al., "Artificial intelligence in medicine," Annals of the Royal College of Surgeons of England, vol. 86, 2004, pp. 334-338.

[4] M. N. O. Sadiku, Y. Zhou, and S. M. Musa, "Natural language processing in healthcare," International Journal of Advanced Research in Computer Science and Software Engineering, vol. 8, no. 5, May 2018, pp. 39-42.

[5] "No longer science fiction, AI and robotics are transforming healthcare,"

https://www.pwc.com/gx/en/industries/healthcare/publications/ai-robotics-new-health/transforming-healthcare.html 
[6] F. Jiang et al., "Artificial intelligence in healthcare: Past, present and future,” Stroke and Vascular Neurology, 2017.

[7] J. Lynden, "What does it mean to 'humanise' technology?" November 2018, https://becominghuman.ai/what-does-it-mean-to-humanise-tech-1c6c4f28bf91

[8] "Differences between artificial intelligence vs human intelligence," https://www.educba.com/artificial-intelligence-vs-human-intelligence/

[9] T. Bandopadhyay and S. Bharani, "Humanized AI for analytics that matter," January 2019, https://www.wipro.com/en-US/analytics/humanized-ai-for-analytics-that-matter/

[10]E. Norton, "The application of humanization theory to health-promoting practice," Perspectives in Public Health, vol. 135, no. 3, May 2015, pp. 133-137.

[11]R. Garza-Hernandez et al., "Surgical patients' perception about behaviours of humanized nursing care," Hispanic Health Care International, July 2019.

[12] "Artificial intelligence examples," http://major.magdalene-project.org/artificial-intelligence-examples/

[13] J. Kite-Powell, "How do we create artificial intelligence that is more human?" https://www.forbes.com/sites/jenniferhicks/2019/03/19/how-do-we-create-artificial-intelligence-that-is-morehuman/\#6787a1d14920

[14] A. Daniele and Y. Z. Son, "AI+Art=Human," Proceedings of the 2019 AAAI/ACM Conference on AI, Ethics, and Society, Honolulu, HI, USA, January 2019, pp. 155-161.

[15] E. Letouzé and A. Pentland, "Towards a human artificial intelligence for human development," ITU Journal: ICT Discoveries, no. 2, December 2018.

[16] E. Topol, Deep Medicine: How Artificial Intelligence Can Make Healthcare Human Again. Basic Books, 2019.

[17] P. R. Daugherty and H. J. Wilson, Human + Machine: Reimagining work in the age of AI. Harvard Business Review Press, 2018.

\section{ABOUT AUTHORS}

Matthew N.O. Sadiku is a professor emeritus in the Department of Electrical and Computer Engineering at Prairie View A\&M University, Prairie View, Texas. He is the author of several books and papers. His areas of research interest include computational electromagnetics and computer networks. He is a fellow of IEEE.

Yogita P. Akhare is a doctoral student at Prairie View A\&M University, Prairie View, Texas. Her research interests include machine drives and nanotechnology.

Abayomi Ajayi-Majebi is a professor in the Department of Manufacturing Engineering at Central State University in Wilberforce, Ohio. In 2015 he was honored by the White House as a Champion of Change for his significant contributions to the engineering education of minority students. He is a senior member of both the Society of Manufacturing Engineers and the American Society for Quality.

Sarhan M. Musa is a professor in the Department of Electrical and Computer Engineering at Prairie View A\&M University, Texas. He has been the director of Prairie View Networking Academy, Texas, since 2004. He is an LTD Sprint and Boeing Welliver Fellow. His areas of research interest include computational electromagnetics and computer networks. 
International Journal of Advances in Scientific Research and Engineering (ijasre), Vol 6 (12), December -2020

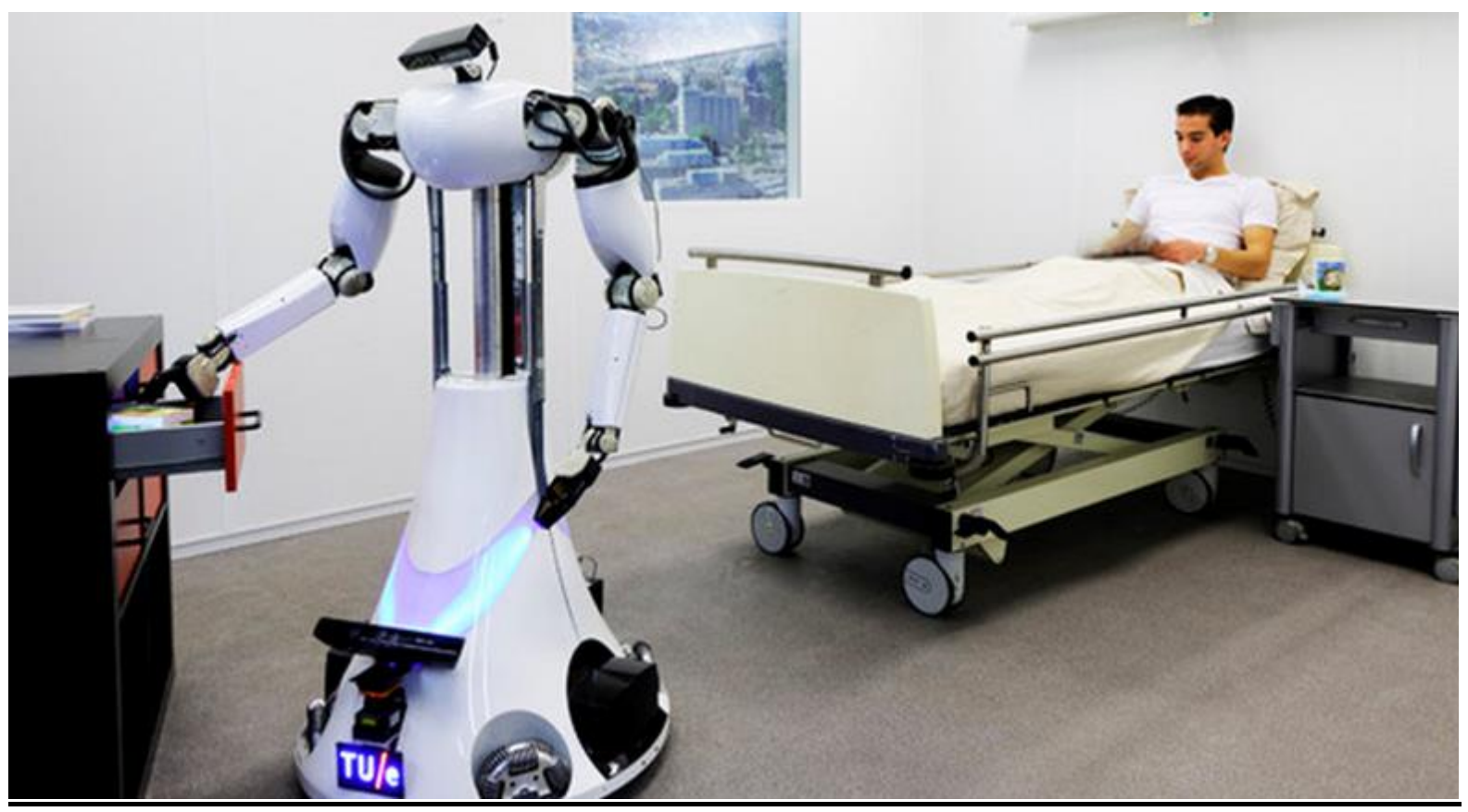

Figure 1: An example of Human-AI system in healthcare [11]. 